Activation of the inflammatory response system and varied levels of cytokines in acute schizophrenia have been suggested by recent studies. Psychopharmacologic agents can differentially effect cytokine production, which suggests that therapeutic function of neuroleptics may involve immunomodulation.

The present study was carried out to examine: (i) serum concentrations of interleukin (IL)-1 $\beta$, soluble interleukin-2 receptor (sIL-2R), IL-6, IL-8 and tumour necrosis factor (TNF)- $\alpha$ in schizophrenic patients; (ii) their relation with psychopathological assessment; and (iii) the relation of the initial cytokine levels with responsiveness to risperidone therapy.

Thirty-four drug-free schizophrenic patients with acute exacerbation and 23 age- and gender-matched healthy controls were recruited for this study. Psychopathological assessments at admission and throughout risperidone treatment for 60 days were recorded. Serum cytokine concentrations were determined with chemilumunescence assays.

According to our results, serum IL-1 $\beta$, sIL-2R, IL-6, IL-8 and TNF- $\alpha$ concentrations adjusted for age, gender, body mass index and smoking were no different in patients with schizophrenia and controls and among subtypes of schizophrenia. However, the initial TNF- $\alpha$ concentrations had a significant effect on Brief Psychiatric Rating Scale and Scale Assessment of Positive Symptoms scores. The initial cytokine concentrations of the patients responsive to risperidone were not significantly different from those of non-responsive patients.

The present study demonstrates that plasma levels of IL-1 $\beta$, sIL-2R, IL-6, IL-8 and TNF- $\alpha$ adjusted for confounding factors are not altered in drug-free schizophrenic patients at acute exacerbation. We suggest that, if cytokine production is altered in schizophrenia, these alterations may not be detectable in systemic circulation. According to our results, the therapeutic effect of risperidone is not related to basal levels of the aforementioned cytokines. However, serum TNF- $\alpha$ may contribute to symptomatology in schizophrenia.

Key words: Interleukins, Schizophrenia, Risperidone, Tumour necrosis factor- $\alpha$

\section{Serum IL-1 $\beta$, sIL-2R, IL-6, IL-8 and TNF- $\alpha$ in schizophrenic patients, relation with symptomatology and responsiveness to risperidone treatment}

\author{
Ayşe Binnur Erbağci ${ }^{\mathrm{CA}}$, Hasan Herken, \\ Oya Köylüoglu, Necat Yilmaz and \\ Mehmet Tarakçioglu
}

Department of Biochemistry and Clinical

Biochemistry, Faculty of Medicine, University of

Gaziantep, TR-27310 Gaziantep, Turkey

\author{
${ }^{\mathrm{CA}}$ Corresponding Author \\ Tel: (+90) 3423601200 \\ Fax: (+90) 3423601617 \\ E-mail: erbagci@gantep.edu.tr
}

\section{Introduction}

Over the past decade, as immunologic, neurochemical and neuroendocrine activities of the cytokines involving the brain and their behavioural effects have been demonstrated, research concerning the role of cytokines in schizophrenia has expanded. ${ }^{1}$ Activation of the inflammatory response system in acute schizophrenia has been suggested by several recent studies. Schizophrenic patients have aberrant proportions of immuno-competent cells and varied levels of cytokines, especially proinflammatory interleukin (IL)-6, IL-1 and tumour necrosis factor (TNF)- $\alpha$, in their peripheral blood or cerebrospinal fluid. ${ }^{2}$ A decrease in mitogen-stimulated IL-2 production by peripheral blood mononuclear cells and elevated levels of soluble interleukin-2 receptor (sIL-2R) were also observed. ${ }^{3,4}$ Theoretically, alterations in the levels of cytokines can simply be a consequence of mental stress or sleep deprivation associated with the onset or exacerbation of the disease. On the contrary, they can modify metabolism of neurotransmitters and neuroendocrine hormones, and influence neural development and neurodegeneration, as supported by findings in fundamental neuroscience. ${ }^{1,2,5}$ 
IL-8, which has not been studied to date in schizophrenia, belongs to the chemoattractant cytokines (namely, the chemokine subfamily) and takes part in immune response, cell adhesion and angiogenesis. $^{6}$

Cytokines, which were discovered as humoral mediators of cell-to-cell interactions within the immune system, have been found to be key factors for the host responses that are mediated by the central nervous system, which include fever and activation of the hypothalamic-pituitary-adrenal system. Behavioural-activating effects of certain cytokines like the somnogenic activity of IL-1 and TNF- $\alpha$ and the suppresser effects of IL-1 $\beta$, IL- 8 and TNF- $\alpha$ on appetite are also demonstrated. ${ }^{1}$ In an animal study, IL-1-, IL-2and IL-6-treated mice displayed significant differences in exploration. ${ }^{7}$ In schizophrenic patients, different patterns of cytokine production and psychotic behaviour such as paranoid behaviour and IL-10 are suggested. ${ }^{8}$ Furthermore, the administration of cytokines may induce numerous psychiatric symptoms such as fatigue, apathy, depressive mood and delusions. ${ }^{1}$ Therefore, it is reasonable to assume that the presumed immunopathology in psychiatric disorders might lead to alterations in circulating cytokine levels.

Although the aetiology, pathogenesis and mechanism of schizophrenia has remained elusive, efficient treatment with neuroleptic medication for acute schizophrenia has been proved beyond doubt. Actions of these drugs and biochemical and immunological changes that occur in response to treatment have advanced our understanding of the basis of schizophrenia. However, $20-40 \%$ of the patients are resistant to standard antipsychotic treatments, suggesting a contribution of different mechanisms in individual patients. ${ }^{9}$ With this point of view, response to a specific therapy may be a tool for understanding pathophysiology of the disease. Risperidone is a benzisoxazole derivate, chemically unrelated to any other currently available antipsychotic drug. Risperidone, like clozapine, is a potent 5-hydroxytryptamine $(5-\mathrm{HT})_{2}$ and relatively weak $\mathrm{D}_{2}$ dopamine receptor antagonist. 9

Although very limited, several studies have reported changes in the levels of specific cytokines in conjunction with psychopharmacologic agents, suggesting that therapeutic action of neuroleptics may involve immunomodulation. In vitro studies have shown that chlorpromazine has an inhibitory effect on the production of IL-2, TNF- $\alpha$ and interferon by human lymphocytes. In humans, neuroleptic treatment has been associated with an increase in the serum levels of sIL-2R and a decrease in the levels of sIL-6R. ${ }^{1,10}$ The immunomodulatory action of risperidone involving the Clara cell protein and sIL-2R has also been suggested. ${ }^{11}$ However, associations between changes in the levels of cytokines and the therapeutic response have not been firmly established.
In this study, serum IL-1 $\beta$, sIL-2R, IL-6, IL-8 and TNF$\alpha$ concentrations of 34 drug-free schizophrenic patients were analysed. Psychopathological assessments at admission and throughout risperidone treatment for 60 days were recorded to evaluate whether cytokine levels have any effect on Brief Psychiatric Rating Scale (BPRS), Scale Assessment of Positive Symptoms (SAPS) and Scale Assessment of Negative Symptoms (SANS) scores, and on improvement achieved with an atypical neuroleptic risperidone.

\section{Material and methods}

\section{Subjects}

The study was conducted at Gaziantep University, Faculty of Medicine (Department of Psychiatry, and Department of Biochemistry and Clinical Biochemistry) in 2000. Informed consent was obtained from all subjects according to the Helsinki declaration as revised in 1996. Thirty-four patients with schizophrenia (15 males and 19 females) were recruited. The mean age was 33.1 years (range, 17-72 years). The mean duration of illness was 5.9 years (range, 1.0-25.0 years). Eleven patients were smokers; none of them were alcohol consuming.

Healthy non-psychiatric controls $(n=23)$ were recruited from the hospital staff. Eight of the controls were male and 15 were female. The mean age was 29.2 years (range, 18-44 years). Fifteen were smokers; none of them were alcohol consuming. None of the healthy controls had a history of psychiatric disorder, drug abuse or dependence, serious medical conditions or seizure disorders.

\section{Protocol}

Prior to admission, patients were treated with typical neuroleptic drugs. After withdrawal of typical neuroleptics for 1 week, blood samples were taken and initial psychopathologic assessment was recorded, followed by treatment with $4 \mathrm{mg}$ daily doses of risperidone for 60 days.

The initial psychopathological assessment (BPRS, SANS, SAPS) at day $1\left(\mathrm{~T}_{1}\right)$ was reviewed at every point of investigation, at day $15\left(\mathrm{~T}_{2}\right)$, at day $30\left(\mathrm{~T}_{3}\right)$ and at day $60\left(\mathrm{~T}_{4}\right)$. All patients were monitored for somatic illness throughout the investigation period and were excluded if symptoms of infections or symptoms of systemic somatic illness were present. Thirty-four patients were included according to these criteria.

\section{Clinical assessment}

A DSM-IV diagnosis of chronic schizophrenia was established on the basis of independent structured clinical interviews and a review of records by a qualified psychiatrist that included the BPRS. ${ }^{12}$ Patients with any form of psychiatric comorbidity including 
Table 1. Characteristics of the study participants

\begin{tabular}{lcc}
\hline & Patients $(n=34)$ & Controls $(n=23)$ \\
\hline Gender $($ male/female) & $15 / 19$ & $8 / 15$ \\
Age $(\text { years })^{\mathrm{a}}$ & $30(17-72)$ & $28(18-44)$ \\
Smoker $(n)$ & $11,32.3 \%$ & $15,65.2 \%$ \\
Body mass index $\left(\mathrm{kg} / \mathrm{m}^{2}\right)^{\mathrm{a}}$ & $23.4(18.3-39.1)$ & $22.3(17.2-39.5)$ \\
\hline
\end{tabular}

${ }^{a}$ Data presented as median (minimum-maximum).

substance abuse were excluded. Sixteen patients met the criteria for paranoid, 14 for residual, three for disorganised and one patient for an undifferentiated form of acute or chronic schizophrenia. SAPS and SANS were also recorded throughout the investigation period. Patients with a $20 \%$ decrease in BPRS, SANS and SAPS at $\mathrm{T}_{4}$ were accepted as responsive to risperidone.

Age of the schizophrenia onset was defined as the age at which symptoms first appeared as reported by the patient, the family or medical staff.

\section{Experimental}

Blood samples were collected using standard venipuncture technique between 9:30 and 11:00 a.m. after $12 \mathrm{~h}$ fast. Serum samples were separated immediately after centrifugation at $+4^{\circ} \mathrm{C}, 2000 \times g$ for $10 \mathrm{~min}$ and stored at $-20^{\circ} \mathrm{C}$ until analysis, which was performed in the same run to avoid inter-run analytical variation. Serum IL-1 $\beta$, sIL-2R, IL-6, IL-8 and TNF- $\alpha$ levels were determined with chemiluminescence enzyme immunometric assays on an Immulite Immunoassay Analyser (Immulite IL-1 $\beta{ }^{\circledR}$, sIL-2R ${ }^{\circledR}$, IL-6 ${ }^{\circledR}$, IL$8{ }^{\circledR}$ and TNF- $\alpha{ }^{\circledR}$ assays; DPC, Los Angeles, CA, USA). The analytical sensitivities of IL-1 $\beta$, sIL-2R, IL-6, IL-8 and TNF- $\alpha$ assays were $1.5 \mathrm{pg} / \mathrm{ml}, 10 \mathrm{U} / \mathrm{ml}, 1.0 \mathrm{pg} / \mathrm{ml}$, $2.0 \mathrm{pg} / \mathrm{ml}$ and $1.7 \mathrm{pg} / \mathrm{ml}$, respectively, and the lower reportable ranges were $5.0 \mathrm{pg} / \mathrm{ml}, 50 \mathrm{U} / \mathrm{ml}, 5.0 \mathrm{pg} / \mathrm{ml}$, $5.0 \mathrm{pg} / \mathrm{ml}$ and $4.0 \mathrm{pg} / \mathrm{ml}$, respectively. The crossreactivities of IL-1 $\alpha$, IL-2, IL-2R, IL-4, IL-5, IL-6, IL-8, IL10 , TNF- $\alpha$ and interferon- $\gamma$ for the IL- $1 \beta$ assay, the cross-reactivities of IL-1 $\beta$, IL-2, IL-6, IL-8, IL-10, TNF- $\alpha$ and interferon- $\gamma$ for the sIL-2R assay, the crossreactivities of IL-1 $\beta$, IL-2, IL-2R, IL-4, IL-6, TNF- $\alpha$ and interferon- $\gamma$ for the IL- 8 assay, and the cross-reactiv- ities of IL-1 $\beta$, IL-2, IL2R, IL-4, IL-6, IL-8, IL-10 and IL-13 for the TNF- $\alpha$ assay were non-detectable. The crossreactivities of IL-1 $\alpha$, IL-1 $\beta$, IL-2, IL- 4 , IL-8, TNF- $\alpha$ and interferon- $\gamma$ for the IL- 6 assay were $\leq 0.065 \%$ as reported by the manufacturer. Immulite cytokine controls were included in each analytical run. Intraassay and inter-assay precision performances of the assays were determined on 10 replicates in a single run and in 20 different runs, respectively, from the quality control data of the laboratory. Coefficients of variation obtained were within the 3.4-8.8\% range.

\section{Statistical}

Data are presented as mean \pm SEM or median (minimum-maximum) when $n \leq 30$. Because IL-1 $\beta$, sIL-2R, IL-6 and IL-8 distribution was skewed rightward, a natural $\log$ transformation was applied to normalise the data for analysis. A general linear model was used to consider case-control differences after adjustment for other factors and covariates on transformed data. The paired-samples $t$-test for psychopathological assessment and the Kruskal-Wallis test was performed for subgroups. Case-control differences in nominal data (gender, smoking) were evaluated with the chi-squared test. Two-tailed $p<0.05$ was considered significant. Analyses and illustrations were performed with SPSS 9.0 (SPSS Inc., Chicago, IL, USA) and MedCalc (Mariakerke, Belgium) statistical software programs.

\section{Results}

The baseline characteristics of the study participants are presented in Table 1. Because of matching criteria,

Table 2. Psychopathological assessments of the patients with schizophrenia throughout the investigation period (mean \pm SEM)

\begin{tabular}{|c|c|c|c|c|}
\hline & $\mathrm{T}_{1}$ (day 1) & $\mathrm{T}_{2}$ (day 15) & $\mathrm{T}_{3}$ (day 30 ) & $\mathrm{T}_{4}$ (day 60 ) \\
\hline $\begin{array}{l}\text { BPRS* } \\
\text { SANS } \\
\text { SAPS* }\end{array}$ & $\begin{array}{l}45.6 \pm 1.3 \\
79.2 \pm 5.7 \\
58.4 \pm 4.4\end{array}$ & $\begin{array}{l}36.2 \pm 1.6 \\
70.1 \pm 5.3 \\
43.7 \pm 3.6\end{array}$ & $\begin{array}{l}28.9 \pm 1.6 \\
58.1 \pm 4.3 \\
32.8 \pm 3.3\end{array}$ & $\begin{array}{l}24.0 \pm 1.8 \\
51.4 \pm 4.0 \\
26.4 \pm 3.2\end{array}$ \\
\hline
\end{tabular}

BPRS, Brief Psychiatric Rating Scale; SAPS, Scale Assessment of Positive Symptoms; SANS, Scale Assessment of Negative Symptoms.

${ }^{*} p<0.001$ for $T_{1}$ versus $T_{2}, T_{2}$ versus $T_{3}, T_{3}$ versus $T_{4}$, and $T_{1}$ versus $T_{4}$.

† $p<0.001$ for $\mathrm{T}_{1}$ versus $\mathrm{T}_{2}, \mathrm{~T}_{2}$ versus $\mathrm{T}_{3}$ and $\mathrm{T}_{1}$ versus $\mathrm{T}_{4} ; p<0.005 \mathrm{~T}_{3}$ versus $\mathrm{T}_{4}$. 
Table 3. Serum median (minimum-maximum) interleukin concentrations of the patients with schizophrenia and the healthy control group at $T_{1}$

\begin{tabular}{lcc}
\hline & Patients $(n=34)$ & Controls $(n=23)$ \\
\hline $\mathrm{slL}-2 \mathrm{R}(\mathrm{U} / \mathrm{ml})$ & $583(262-3567)$ & $625(303-858)$ \\
$\mathrm{IL}-1 \beta(\mathrm{pg} / \mathrm{ml})$ & $5.0(5.0-11.6)$ & $5.0(5.0-5.0)$ \\
$\mathrm{IL}-6(\mathrm{pg} / \mathrm{ml})$ & $5.0(5.0-13.2)$ & $5.0(5.0-7.7)$ \\
$\mathrm{IL}-8(\mathrm{pg} / \mathrm{ml})$ & $10.2(5.0-1683.0)$ & $8.5(5.0-233.0)$ \\
$\mathrm{TNF}-\alpha(\mathrm{pg} / \mathrm{ml})$ & $6.3(4.0-10.9)$ & $6.8(4.0-14.1)$ \\
\hline
\end{tabular}

sIL-2R, Soluble interleukin-2 receptor; IL, interleukin; TNF, tumour necrosis factor.

no statistically significant difference was noted in age, gender, smoking and body mass index (BMI).

\section{Psychopathology}

At admission $\left(\mathrm{T}_{1}\right)$, the BPRS, SANS and SAPS scores were (mean \pm SEM) $45.6 \pm 1.3,79.2 \pm 5.7$ and $58.4 \pm$ 4.4, respectively. During 60 days of in-patient treatment, BPRS, SANS and SAPS scores improved significantly from $T_{1}$ to $T_{2}$, from $T_{2}$ to $T_{3}$, and from $T_{3}$ to $\mathrm{T}_{4}$ (Table 2). Total improvements ( $\mathrm{T}_{1}$ to $\mathrm{T}_{4}$ ) of the scores were $46.2 \pm 4.3,33.0 \pm 4.0$ and $53.0 \pm 5.1 \%(p$ $<0.001$ ), respectively.

\section{Immunological findings}

Plasma interleukin levels were measured in patients with schizophrenia at $T_{1}$ and compared with the controls. A statistically significant difference was not observed for serum $\log$ sIL-2R, $\log$ IL-1 $\beta, \log$ IL- $6, \log$ IL-8 and TNF- $\alpha$ adjusted for age, gender, smoking and BMI (Table 3 and Figs. 1-3). A significant intercorrelation between TNF- $\alpha$ and IL-8 was detected only in the patient group $(r=0.486, p<0.006)$.

Plasma interleukin levels $\left(\mathrm{T}_{1}\right)$ were not different among subtypes of schizophrenia $(\phi>0.05)$ (Table 4).

\section{Nominal data and cytokines}

In the case group, no significant correlation was seen between cytokine concentrations and age, BMI and duration of the disease, and no difference was observed in relation to gender, smoking, positive family history and education.

\section{Relation of psychopathology and cytokines}

Regarding psychopathological assessment at $\mathrm{T}_{1}$, only TNF- $\alpha$ concentrations had a significant effect on BPRS and SAPS according to the general linear model that included age, smoking, BMI, gender and subtypes of schizophrenia $(p=0.019$ and $p=$ 0.030) (Table 5).

\section{Responsiveness to risperidone and cytokines}

The initial cytokine concentrations of the patients responsive to risperidone were not significantly different from those of non-responsive patients.

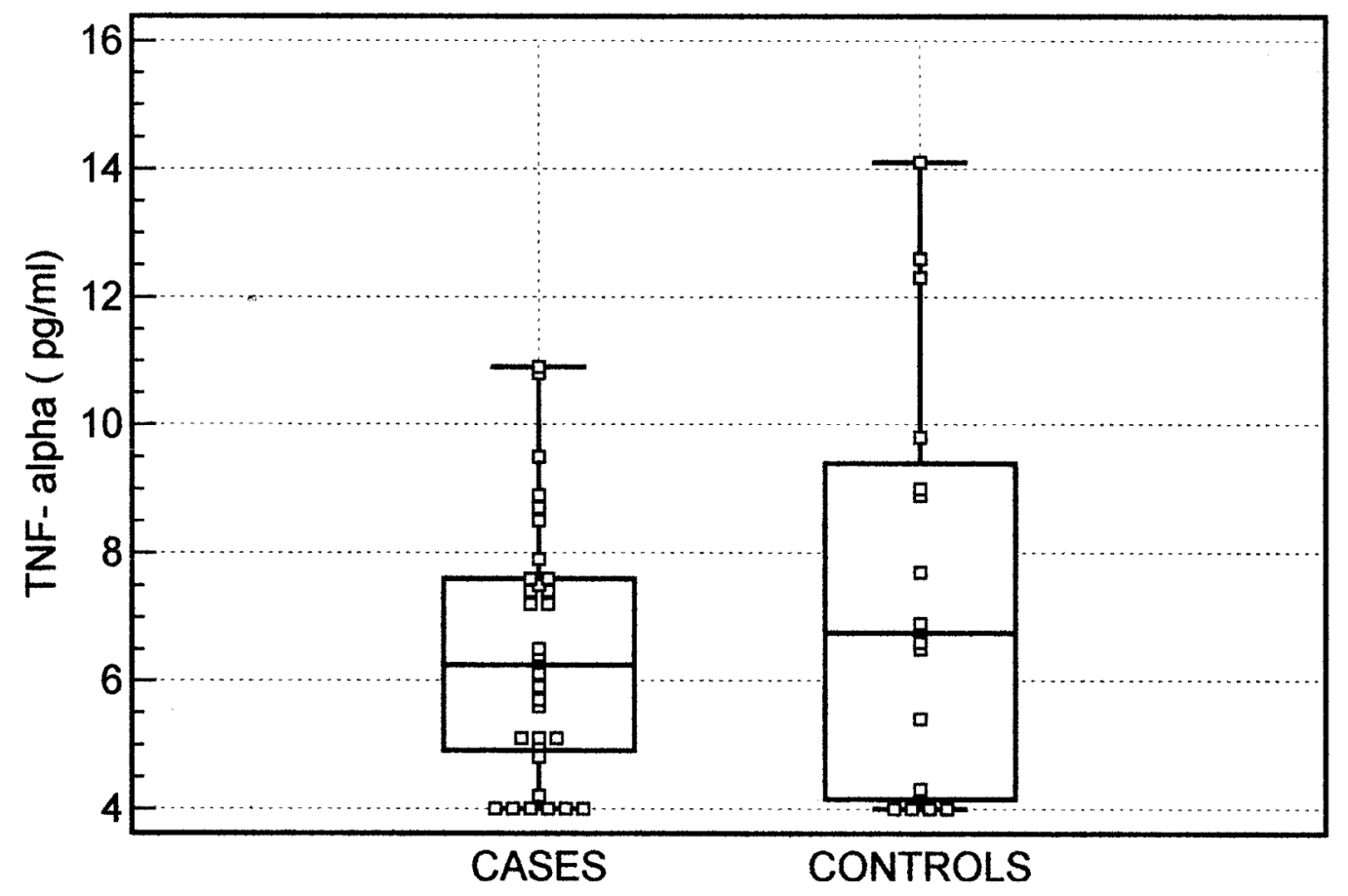

FIG. 1. Serum tumour necrosis factor-alpha (TNF- $\alpha$ ) concentrations in the schizophrenic patients and the healthy controls. The central box represents the values from the 25 th to 75 th percentiles. The middle line represents the median. A line extends from the minimum to the maximum values, excluding extreme values. 


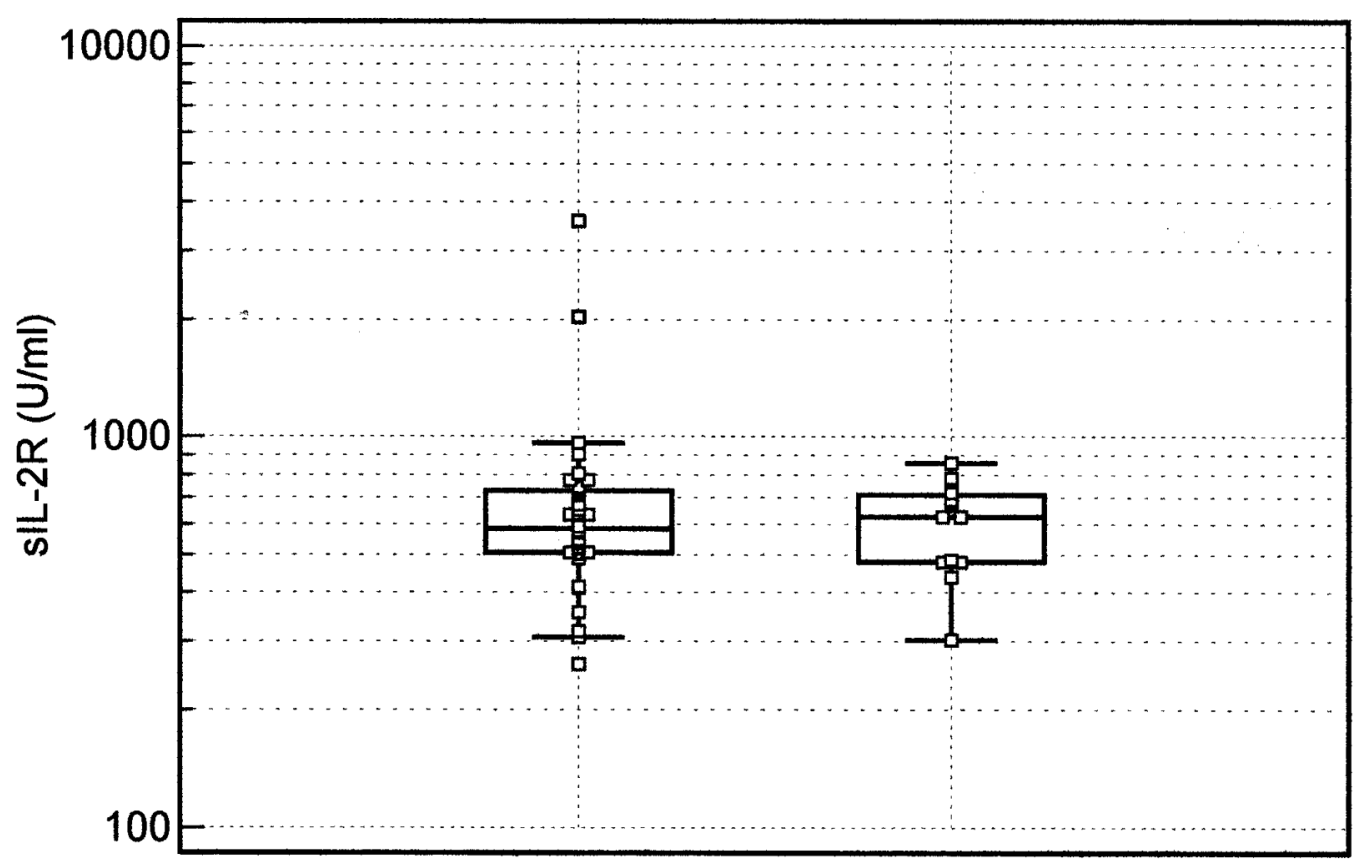

CASES

CONTROLS

FIG. 2. Serum soluble interleukin-2 receptor (sIL-2R) concentrations in the schizophrenic patients and the healthy controls on a semi-logarithmic scale. The central box represents the values from the 25th to 75th percentiles. The middle line represents the median. A line extends from the minimum to the maximum values, excluding extreme values.

\section{Discussion}

In the present study, a significant difference of IL-1 $\beta$, sIL-2R, IL-6, IL-8 and TNF- $\alpha$ was not observed in schizophrenic patients compared with the healthy controls. Although investigators reported altered circulating levels and in vitro production of cytokines in schizophrenia, the respective evidence is not consistent. Several studies have found a decrease in mitogen-stimulated IL-2 production by peripheral

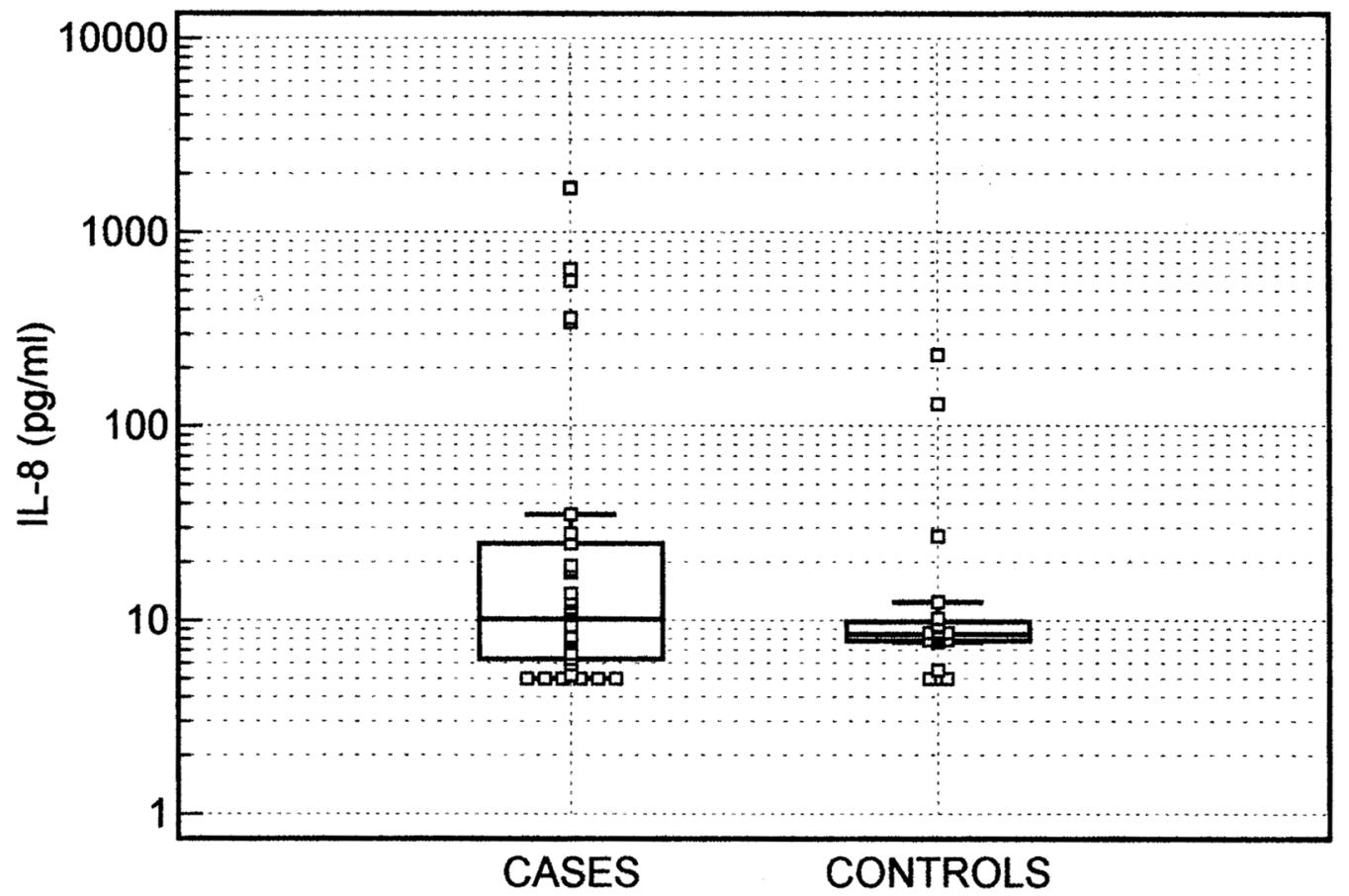

FIG. 3. Serum interleukin (IL)-8 concentrations in the schizophrenic patients and the healthy controls on a semi-logarithmic scale. The central box represents the values from the 25th to 75th percentiles. The middle line represents the median. A line extends from the minimum to the maximum values, excluding extreme values. 
Table 4. Serum median (minimum-maximum) interleukin concentrations of the patients with different subtypes of schizophrenia

\begin{tabular}{lllcc}
\hline & Paranoid $(n=16)$ & Residual $(n=14)$ & Disorganised $(n=3)$ & Controls $(n=23)$ \\
\hline sIL-2R $(\mathrm{U} / \mathrm{ml})$ & $604(307-2030)$ & $642(262-3567)$ & $545(410-590)$ & $625(303-858)$ \\
IL-1 $\beta(\mathrm{pg} / \mathrm{ml})$ & $5.0(5.0-6.0)$ & $5.0(5.0-11.6)$ & $5.0(5.0-5.2)$ & $5.0(5.0-5.0)$ \\
IL-6 $(\mathrm{pg} / \mathrm{ml})$ & $5.0(5.0-13.2)$ & $5.0(5.0-5.0)$ & $5.0(5.0-5.0)$ & $5.0(5.0-7.7)$ \\
IL-8 $(\mathrm{pg} / \mathrm{ml})$ & $8.1(5.0-647.0)$ & $9.3(5.0-1683.0)$ & $27.8(19.1-362.0)$ & $8.5(5.1-233.0)$ \\
TNF- $\alpha(\mathrm{pg} / \mathrm{ml})$ & $6.1(4.0-10.8)$ & $6.7(4.0-9.5)$ & $5.1(4.0-10.9)$ & $6.8(4.0-14.1)$ \\
\hline
\end{tabular}

sIL-2R, Soluble interleukin-2 receptor; IL, interleukin; TNF, tumour necrosis factor.

blood mononuclear cells, which is the most frequently confirmed finding in the immunology of schizophrenia. ${ }^{3,13-15}$ Elevated levels of the sIL-2R in peripheral blood, a sign that also indicates lymphocyte activation, were also repeatedly observed in schizophrenia, ${ }^{4,16,17}$ whereas other studies failed to find differences between patients and healthy controls. ${ }^{10,18,19}$ Moreover, circulating levels of other leukocyte activation markers such as SCD14 and SCD23 were not higher than controls. ${ }^{20}$ Other frequently replicated immune alterations include an increase in serum IL-6 concentrations in schizophrenia. $^{21,22}$ However, some studies reported no difference or even decreased IL-6 levels in drug-free patients. $^{23-27}$ Results regarding levels and/or production of IL- 1 and TNF- $\alpha$ have been inconclusive, and are reported to be increased, decreased or unchanged. ${ }^{18,20,25,26,28}$ Haack et al. have shown that when confounding factors like age, gender, BMI, smoking habits and prior medication are taken into account, plasma levels of IL-6, TNF- $\alpha$ and sIL-2R are not different. $^{29}$ Our results that show no difference in circulating IL-1 $\beta$, sIL-2R, IL- 6 , IL- 8 and TNF- $\alpha$ concentrations adjusted for age, gender and smoking in drugfree schizophrenic patients confirm this previous study. However these findings do not definitively exclude a pathophysiological role for cytokines in schizophrenia because they effect primarily the brain where the regulation of cytokine production differs considerably from the periphery. However, the present study suggests that if cytokine production is altered in schizophrenia, these alterations may not be detectable in the systemic circulation.

The second major finding of this study is that TNF- $\alpha$ may be associated with symptomatology of schizo- phrenia: BPRS and SAPS. Although part of the response of the organism to an infection or injury is local and occurs at the cellular and molecular levels, another part occurs at the level of the organism and involves specific adaptive behaviours such as increased sleep, decreased appetite and sexual drive, which may be at least partly attributed to specific effects of cytokines. There is growing evidence that TNF- $\alpha$ may be associated with somnolence and anorexia. Additionally, the stimulatory effect of TNF- $\alpha$ on hypothalamic-pituitary-adrenal activity, which is a feedback mechanism to suppress an otherwise exaggerated immune/inflammatory response, could effect symptomatology on a disturbed neurochemical basis in schizophrenia. ${ }^{1}$ Naudin et al. previously found higher levels of TNF- $\alpha$ in schizophrenic patients with no relation between global, negative and positive subscale scores. However, in that study, a small proportion of patients who had high TNF- $\alpha$ levels also had elevated IL-6 levels, suggesting a systemic cause for elevation of these proinflammatory cytokines, which in turn may lead to discordance of psychopathological assessment and TNF- $\alpha .^{20}$

In the present study, a relation between improvement of psychopathological assessment during risperidone treatment and the initial cytokine levels was not observed. Previously, an immunosuppresive effect of neuroleptics through suppression of IL-6- or IL6R-related mechanisms, ${ }^{29}$ clozapine-induced increments in plasma sCD8, IL-6, CC16, IL-1RA 1 , sTNF-R2 and sIL-2R concentrations, ${ }^{30,31}$ and increased serum sIL-2R and decreased sIL-6R levels ${ }^{1}$ were reported, whereas no differences in IL- 2 and IFN- $\gamma$ production between unmedicated and medicated schizophrenics $^{32}$ were observed. Chronic treatment with risper-

Table 5. Significant relations of TNF- $\alpha$ and psychopathological assessments at $T_{1}$

\begin{tabular}{|c|c|c|c|c|}
\hline \multirow[t]{2}{*}{ ANCOVA } & \multicolumn{2}{|c|}{$\operatorname{BPRS}\left(\mathrm{T}_{1}\right)$} & \multicolumn{2}{|c|}{$\operatorname{SAPS}\left(T_{1}\right)$} \\
\hline & $F$ & $\mathrm{p}$ & $\mathrm{F}$ & $p$ \\
\hline TNF- $\alpha$ & 7.41 & 0.019 & 6.10 & 0.030 \\
\hline
\end{tabular}

BPRS, Brief Psychiatric Rating Scale; SAPS, Scale Assessment of Positive Symptoms. 
idone was reported to normalise lower plasma Clara cell protein and increase plasma sIL-2R in a crosssectional study. ${ }^{11}$ Thus, the evidence that antipsychotic agents may modulate cytokines in schizophrenia is contradictory. Our study does not include cytokine levels after treatment but demonstrates that the initial cytokine levels are not indicative of patients responsive to risperidone treatment, suggesting that therapeutic action of risperidone may not involve cytokines investigated.

The present study has some methodological limitations that might contribute to the fact that we did not find a major impact of schizophrenia on IL-6 and IL-1 $\beta$ levels. The majority of the patients and the control group had lower plasma levels than the detection limit of the assays, which raises the possibility that group differences might be present below the detection limit. This possibility is supported by studies using more sensitive IL- 6 and IL-1 $\beta$ assays and reporting increased levels, although other studies using sensitive assays found no differences between schizophrenic cases and controls. ${ }^{24}$ It is also noteworthy that studies reporting differences for IL levels had quite large standard deviations, suggesting considerable heterogeneity in the levels of individual patients. ${ }^{10,21,22,24}$ Therefore, immune alterations may be limited to a special subset of patients.

In summary, the present study demonstrates that circulating levels of cytokines are no different in patients with schizophrenia when confounding factors like age, gender, smoking and BMI are taken into account. Immune alterations observed in the previous studies may indicate a special subset of patients or simply result from confounding factors, medications or somatic causes. Therefore, we conclude that plasma IL-1 $\beta$, sIL-2R, IL-6, IL-8 and TNF- $\alpha$ concentrations yield little, if any, evidence for immunopathology in schizophrenia. However, TNF- $\alpha$ may contribute to schizophrenic symptomatology. According to our results, cytokine levels are not indicative of a special subset of patients responsive to risperidone.

ACKNOWLEDGEMENTS. The authors gratefully acknowledge the excellent technical assistance of Mustafa Dağdeviren and Yakup Tuncel.

\section{References}

1. Kronfol Z, Remick DG. Cytokines and the brain: implications for clinical psychiatry. Am J Psychiatry 2000; 157: 683-694.

2. Katila H, Hänninen $\mathrm{K}$, Hurme M. Polymorphisms of the interleukin-1 gene complex in schizophrenia. Mol Psychiatry 1999; 4: 179-181.

3. Arolt V, Rothermundt M, Wandinger KP, Kirchner H. Decreased in vitro production of interferon-gamma and interleukin-2 in whole blood of patients with schizophrenia during treatment. Mol Psychiatry 2000; 5:150-158.

4. Gaughran F, O'Neill E, Cole M, Collins K, Daly RJ, Shanahan F. Increased soluble interleukin 2 receptor levels in schizophrenia. Schizophr Res 1998; 29: 263-267.

5. Prolo P, Licinio J. Cytokines in affective disorders and schizophrenia: new clinical and genetic findings. Mol Psychiatry 1999; 4: 109-111.

6. Santos-Rosa M, Bienvenu J, Whicher J. Cytokines. In: Burtis CA, Ashwood ER, eds. Tietz Textbook of Clinical Chemistry. London: Saunders Company, 1999:541-617.
7. Zalcman S, Murray L, Dyck DG, Greenberg AH, Nance DM. Interleukin-2 and -6 induce behavioral-activating effects in mice. Brain Res 1998; 811: 111-121.

8. Cazzullo CL, Scarone S, Grassi B, et al. Cytokines production in chronic schizophrenia patients with or without paranoid behaviour. Prog Neuropsychopharmacol Biol Psychiatry 1998; 22: 947-957.

9. Schulz SC, Buckley PF. Treatment-resistant schizophrenia. In: Hirsch SR, Weinberger DR, eds Schizophrenia. London: Blackwell Science, 1995:469-484.

10. Muller N, Empl M, RiedelM, SchwarzM, Ackenheil M. Neuroleptic treatment increases soluble IL-2 receptors and decreases soluble IL-6 receptors in schizophrenia. Eur Arch Psychiatry Clin Neurosci 1997; 247: 308-313.

11. Maes M, Bosmans E, Ranjan R, et al. Lower plasma CC16, a natural antiinflammatory protein, and increased plasma interleukin-1 receptor antagonist in schizophrenia: effects of antipsychotic drugs. Schizophr Res 1996; 21 : 39-50.

12. Overal JE, Gorhan DR. The brief psychiatric rating scale. Psychol Reports 1962; 10: 799-812.

13. Ganguli R, Rabin BS, Belle SH. Decreased interleukin-2 production in schizophrenic patients. Biol Psychiatry 1989; 26: 424-427.

14. Ganguli R, Brar JS, Chengappa KR, et al. Mitogen-stimulated interleukin-2 production in never-medicated, first-episode schizophrenic patients. The influence of age at onset and negative symptoms. Arch Gen Psychiatry 1995; 52: 668-672.

15. Villemain F, Chatenoud L, Galinowski A, et al. Aberrant T cell-mediated immunity in untreated schizophrenic patients: deficient interleukin-2 production. Am J Psychiatry 1989; 146: 609-616.

16. Ganguli R, Rabin BS. Increased serum interleukin 2 receptor concentration in schizophrenic and brain-damaged subjects. Arch Gen Psychiatry 1989; 46: 292.

17. Maes M, Meltzer HY, Buckley P, Bosmans E. Plasma-soluble interleukin-2 and transferrin receptor in schizophrenia and major depression. Eur Arch Psychiatr Clin Neurosci 1995; 244: 325-329.

18. Baker I, Masserano J, Wyatt RJ. Serum cytokine concentrations in patients with schizophrenia. Schizophr Res 1996; 20: 199-203.

19. Wilke I, Arolt V, Rothermundt M, Weitzsch Ch, Hornberg M, Kirchner H. Investigations of cytokine production in whole blood cultures of paranoid and residual schizophrenic patients. Eur Arch Psychiatr Clin Neurosci 1996; 246: 279-284.

20. Naudin J, Capo C, Giusano B, Mege JL, Azorin JM. A differential role for interleukin-6 and tumor necrosis factor- $\alpha$ in schizophrenia? Schizophr Res 1997; 26: 227-233.

21. Van Kammen DP, McAllister-Sistilli CG, Kelley ME, Gurklis JA, Yao JK. Elevated interleukin-6 in schizophrenia. Psychiatr Res 1999; 87 129-136.

22. Lin A, Kenis G, Bignotti S, et al. The inflammatory response system in treatment-resistant schizophrenia: increased serum interleukin-6. Schizophr Res 1998; 32: 9-15.

23. Kim YK, Kim L, Lee MS. Relationships between interleukins, neurotransmitters and psychopathology in drug-free male schizophrenics. Schizophr Res 2000; 44: 165-175.

24. Shintani F, Kanba S, Maruo N, et al. Serum interleukin-6 in schizophrenic patients. Life Sci 1991; 49: 661-664.

25. Xu HM, Wei J, Hemmings GP. Changes of plasma concentrations of interleukin- $1 \alpha$ and interleukin- 6 with neuroleptic treatment for schizophrenia. Br J Psychiatry 1994; 164: 251-253.

26. Barak V, Barak Y, Levine J, Nisman B, Roisman I. Changes in interleukin-1 $\beta$ and soluble interleukin-2 receptor levels in CSF and serum of schizophrenic patients. J Basic Clin Physiol Pharmacol 1995; 6: 61-69.

27. Wei J, Xu HM, Davies J, Ramchand $\mathrm{CN}$, Ramchand R, Hemmings GP. Interleukins 1,2 and 6 in the plasma of neuroleptic-free and treated schizophrenic patients and their relatives. Schizophr Res 1992; 6: 140.

28. Monteleone P, Fabrazzo M, Tortorella A, Maj M. Plasma levels of interleukin-6 and tumor necrosis factor alpha in chronic schizophrenia: effects of clozapine treatment. Psychiatr Res 1997; 71: 11-17.

29. Maes M, Bosmans E, Calabrese J, Smith R, Meltzer HY. Interleukin-2 and interleukin-6 in schizophrenia and mania: effects of neuroleptics and mood stabilisers. J Psychiatr Res 1995; 29:141-152.

30. Haack M, Hinze-Selch T, Fenzel T, Kraus T, Kühn M, Schuld A, Pollmächer T. Plasma levels of cytokines and soluble cytokine receptors in psychiatric patients upon hospital admission: effects of confounding factors and diagnosis. J Psychiatr Res 1999; 33: 407-418.

31. Maes M, Bosmans $\mathrm{E}$, Kenis $\mathrm{G}$, De Jong R. In vivo immunomodulatory effects of clozapine in schizophrenia. Schizophr Res 1997; 26: 221-225.

32. Rothermundt M, Arolt V, Leadbeater J, Peters M, Rundolf S, Kirchner H Cytokine production in unmedicated and treated schizophrenic patients Neuroreport 2000; 11: 3385-3388.

Received 13 March 2001;

Accepted 10 April 2001 


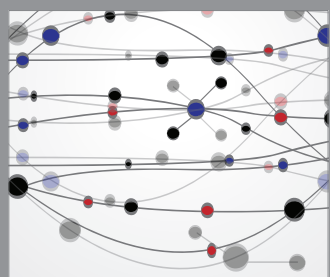

The Scientific World Journal
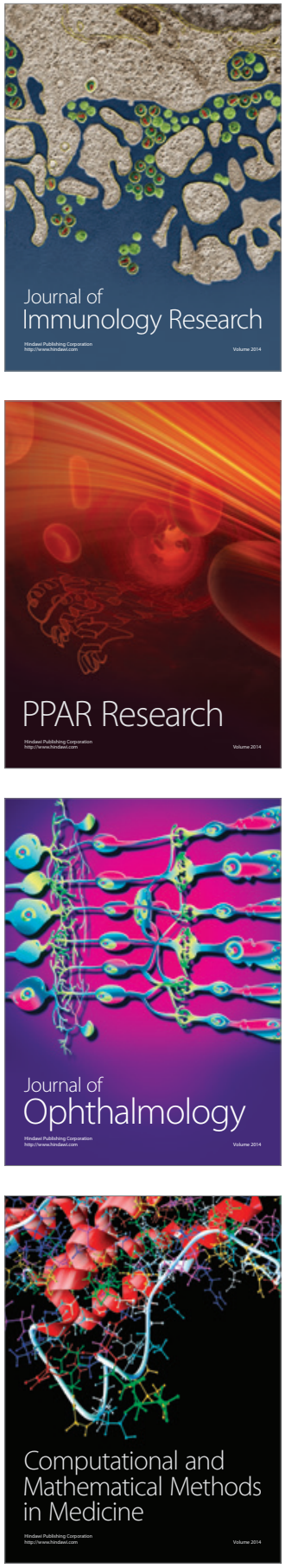

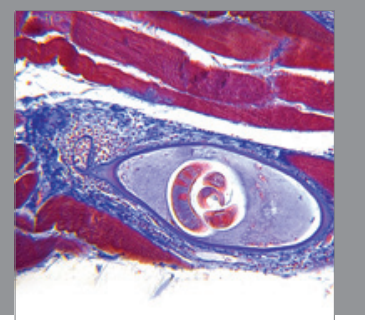

Gastroenterology

Research and Practice
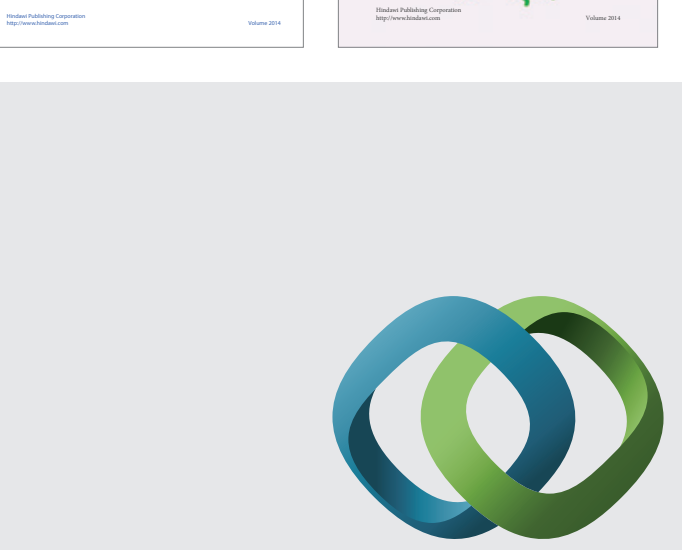

\section{Hindawi}

Submit your manuscripts at

http://www.hindawi.com
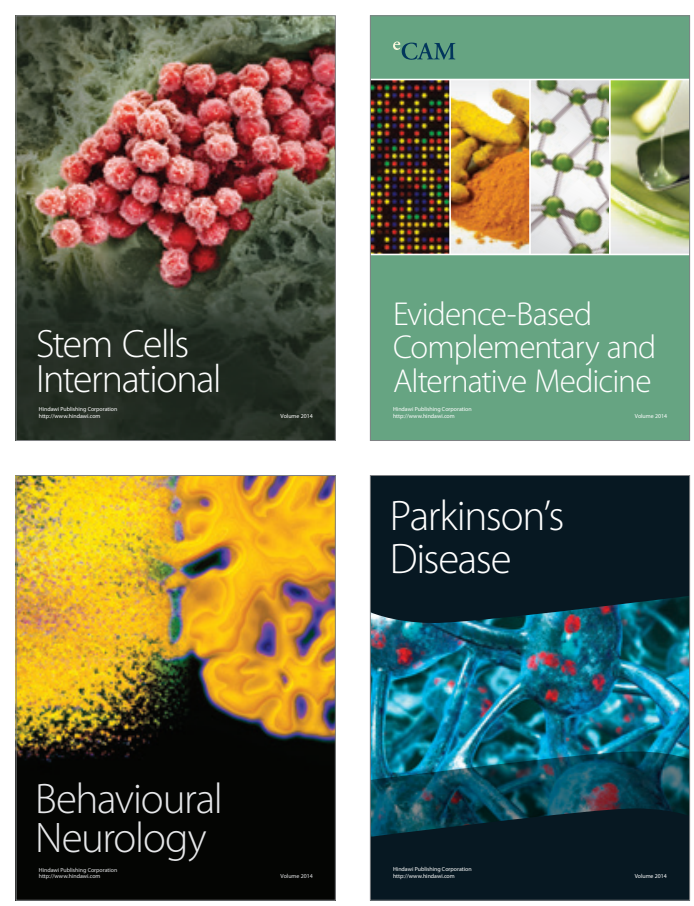

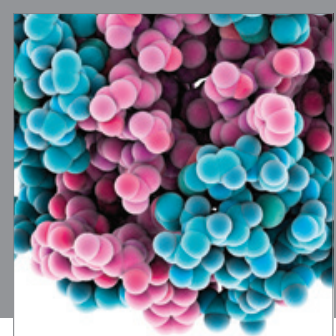

Journal of
Diabetes Research

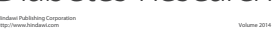

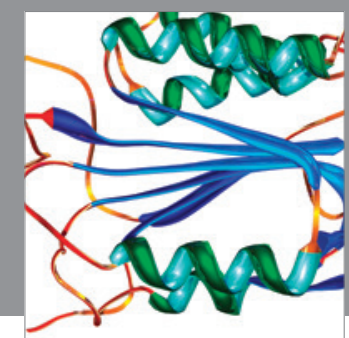

Disease Markers
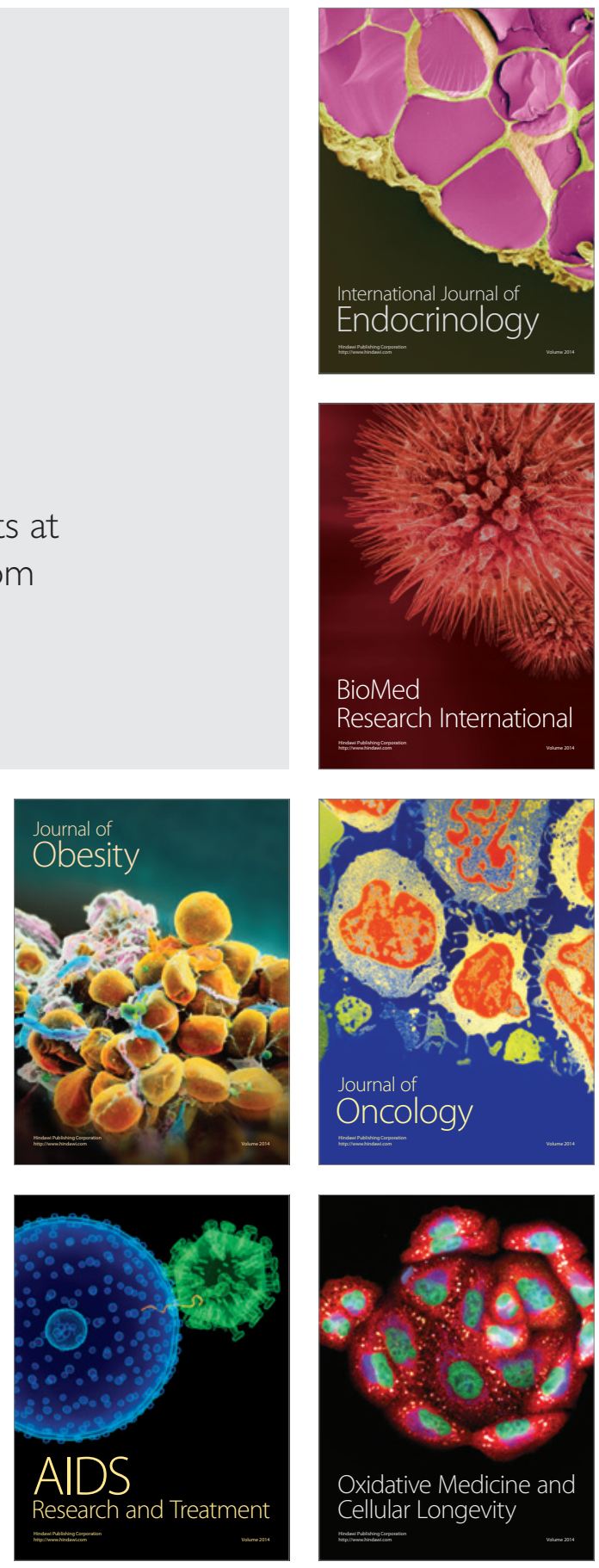